\section{Precision and Detection Limits for EDS Analysis in the SEM}

Eric Lifshin, University at Albany, Albany, NY elifshin@uamail.albany.edu

Raynald Gauvin, McGill University, Montreal, Quebec, raynald.gauvin@McGill.ca

Microanalysts using an EDS on an SEM are sometimes asked whether two samples are of different composition, or if different regions in the same sample vary in composition. A best educated guess may be that differences of a few percent can be distinguished under optimized conditions that include favorable data collection times and count rates, stable instrument operation, and well prepared samples and standards that remain free of contamination. Another frequently asked question is whether it is possible to detect a given amount of a particular element in a sample. The response is that a concentration greater than a few weight percent can generally be detected. Under certain circumstances, detection levels of a few tenths of a weight percent or less are possible. The answer may then be further quali ed by mentioning that the situation can be far worse if there are serious peak overlaps or if the elements of interest are of very low atomic number or both. Such "ball park" estimates are usually based on our experience level with similar samples or the experience of others shared in the literature or elsewhere.

The subject of detection limits and precision relating to $x$-ray microanalysis have been discussed in the literature for some time (1-3). Such estimates are generally based on models in which precision is determined by $x$-ray counting statistics. $X$-ray counting statistics refers to the variability of the number of $x$-ray photons counted in repeated experiments in which the experimental conditions remain constant. Consider a series of SEM-EDS measurements of a pure copper sample made with a beam energy of 20 $\mathrm{keV}$ and a beam current of $1 \mathrm{nA}$ for 10 seconds. The result will be a distribution of values for different 10 second intervals, even if there are no changes in the instrument or the sample from one interval to the next. Why this happens has to do with the inherent variability of the $x$-ray generation process itself in a way that is similar to what is observed for radioactive decay. A plot of the frequency of occurrence of measured values versus those measured values is described by a Poisson distribution although, it is often approximated by a bell shaped or Gaussian curve centered around a mean value of counts $\bar{N}$ and with a standard deviation equal to the $\bar{N}^{1 / 3}$. It is the mean of this distribution that we need to perform accurate quantitative analysis upon, even though analyses are often made on the basis of a single measurement that is just one sample from the distribution of possible results. The situation is actually more complicated because, in quantitative analysis, it is the $K$-ratio that is converted into composition by ZAF or $(\mathrm{z})$ methods where:

$$
K=\frac{\bar{N}-\bar{N}(B)}{\bar{N}_{S}-\bar{N}_{S}(B)}
$$

Four measurements, each with its own variability are involved. $\bar{N}$, the average number of counts measured on the sample, $\bar{N}(B)$ the corresponding average background, $\bar{N}_{S}$ the number of counts on the standard and $\bar{N}_{S}(B)$ the corresponding background on the standard.

Therefore, the question to ask is what is the precision of a given $K$-ratio, rather than an individual measurement, and better yet, what is the precision of the value of the composition, $C$, of a given element determined with that $K$-ratio? Although the equation appears to be rarely used, the answer can be found in an intermediate calculation in Ziebold's classic paper (1) on determining detection limits where the standard deviation in composition is given by:

$$
\sigma_{c}^{2}=C^{2}\left[\frac{\bar{N}+\bar{N}(B)}{n(\bar{N}-\bar{N}(B))^{2}}+\frac{\bar{N}_{s}+\bar{N}_{s}(B)}{n^{\prime}\left(\bar{N}_{s}-\bar{N}_{s}(B)\right)^{2}}\right]\left[1-\frac{(a-1) C}{a}\right]^{2}
$$

where $n$ and $n^{\prime}$ refer to the number of repeat measurements on the sample and standard respectively. The parameter " $a$ " refers to the constant in the Ziebold-Ogilvie (4) equation:

$$
\frac{1-K}{K}=a \frac{1-C}{C}
$$

It can either be determined from standards, or more practically by using either ZAF or ( $z$ ) methods, to calculate $K$ for a given $C$ and then solving equation (3) for " $a$ ". Generally, when an analysis is done, $C$ is determined for an average measured value of $K$ and so it is a relatively easy manner to solve for " $a$ " and then determine

by equation (2). Once is calculated, con dence intervals can be stated when presenting data, e.g. $\mathrm{C} \pm 1.96$ de res the $95 \%$ con dence interval. As an example, gure la shows portions of the low energy $x$-ray spectra of $\mathrm{Al}_{33} \mathrm{Ga}_{67} \mathrm{As}$ and also for an $\mathrm{Al}$ standard indicating the number of counts required for calculating . Data of this type can be obtained from experimental measurements, or in this case, Monte Carlo calculations using the X-ray spectrum simula-
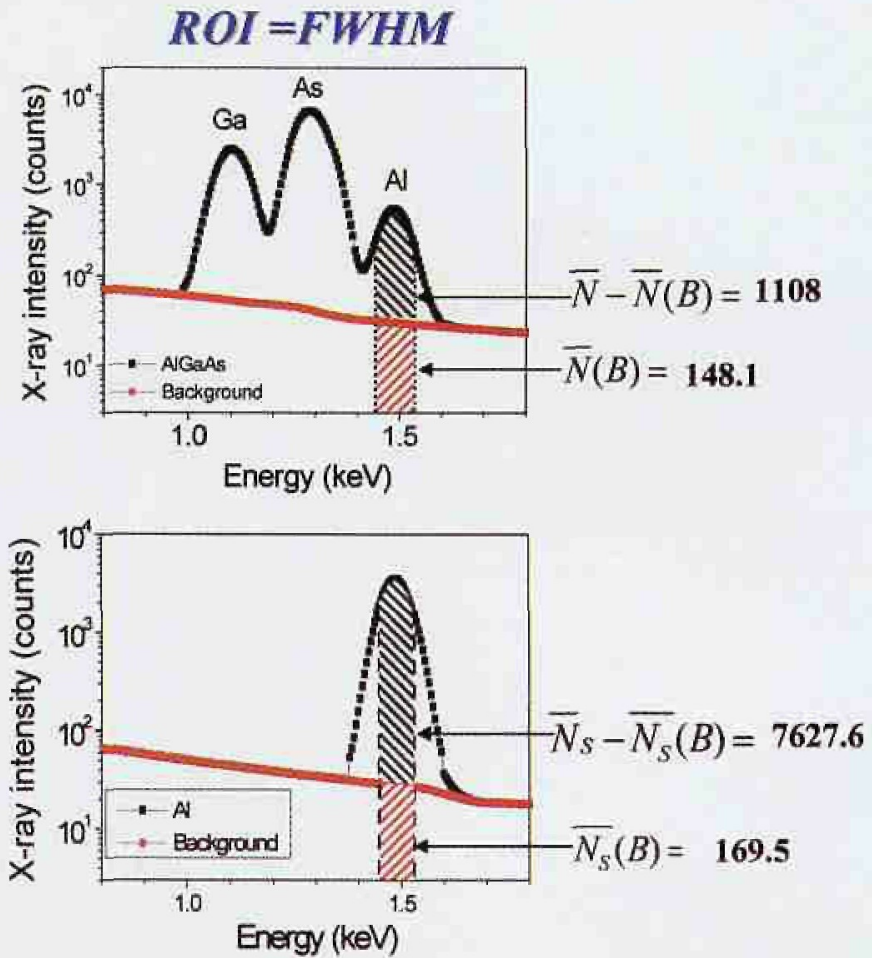

Figure 1a. Monte Carlo Simulation of Spectra for $\mathrm{Al}_{33} \mathrm{Ga}_{67}$. As Sample and pure Al Standard. Conditions: $5 \mathrm{keV}$ beam energy, $1 \mathrm{nA}$ beam current, 10 repeat measurements, $n=n^{\prime}=10$ repeats, 10 second counting intervals

tion program of Gauvin and Lifshin (5), which computes entire x-ray spectra for any well de ned set of operating conditions and detector characteristics. Figure $1 \mathrm{~b}$ shows the quantity $2 / C$ plotted as a function of beam energy. The parameter $2, C$ is approximately $1 / 2$ the $95 \%$ con dence interval normalized by the composition. In this case the concentration of $\mathrm{Al}$ was determined to be 15.2 weight percent with a $95 \%$ con dence interval of $\pm 0.025 \times 15.2= \pm 0.38$ weight percent, when measured under the conditions given at $5 \mathrm{keV}$. This value of precision, if obtainable in practice, would be excellent, but is based solely on counting statistics and does not consider other potential sources of variability such as sample and standard position reproducibility. 


\section{Microscopy Microanalysis}

Table of Contents Preview

Volume 9 , Number 5, October 2003

Special Issue on Electron Diffraction

- Introduction: A Special Issue on Electron Diffraction John G.H. Spence

- Quantitative Convergent Beam Electron Diffraction Measurements of Low-Order Structure Factors in Copper

J. Friis, B. Jiang, J. C.H. Spence, and R. Holmestad

- Analysis of Local Strain in Aluminum Interconnects by Convergent Beam Electron Diffraction

Stephan Krämer, Cynthia A. Volkert, and Joachim Mayer

- Suf cient Conditions for Direct Methods with Swift Electrons L.D. Marks and W. Sinkier

- Quantitative Zone-Axis Convergent Beam Electron Diffraction: Current Status and Future Prospects

Martin Saunders

- A Combination Method of Charge Density Measurement in Hard Materials Using Accurate, Quantitative Electron and X-ray Diffraction: The $\alpha \cdot \mathrm{Al}_{2} \mathrm{O}_{3}$ Case

V. A. Streltsov, P. N.H. Nakashima, and A. W.S. Johnson

- Low-Dose, Low-Temperature Convergent-Beam Electron Diffraction and Multiwavelength Analysis of Hydrocarbon Films by Electron Diffraction

Jinsong Wu and John C.H. Spence

- Accurate Measurements of Valence Electron Distribution and Interfacial Lattice Displacement Using Quantitative Electron Diffraction

Yimei Zhu. Lijun Wu, and J. Tafto

- On the Consistency of QCBED Structure Factor Measurements for TiO, (Rutile)

Bin Jiang, JianMin Zuo, Jesper Friis, and John C.H. Spence

- A Quantitative Nanodiffraction System for Ultrahigh Vacuum Scanning Transmission Electron Microscopy

Gary G. Hembree, Christoph Koch, and John C.H. Spence

- Quantitative Electron Diffraction Evidence for One-Dimensional Ordering in Magnetite above the Verwey Transition

J. Pacaud, J.M. Zuo. R. Hoier, and S. Matsumura

Book Review

- Review of Scanning Electron Microscopy and X-Ray Microanalysis by J. Goldstein, D. Newbury, D. Jay, C. Lyman, P. Echlin, P. Lifshin, L. Sawyer, and J. Michael Sandy Burany

Indexed in Chemical Abstracts, Current Contents, BIOSIS, and MEDLINE (Publlied)

MSA members receive both Microscopy Today and Microscopy and Microanalysis FREE!

\section{JOKE:}

Two hydrogen atoms walk into a bar. One says, "I've lost my electron."

The other says, "Are you sure?"

The first replies, "Yes, I'm positive..."

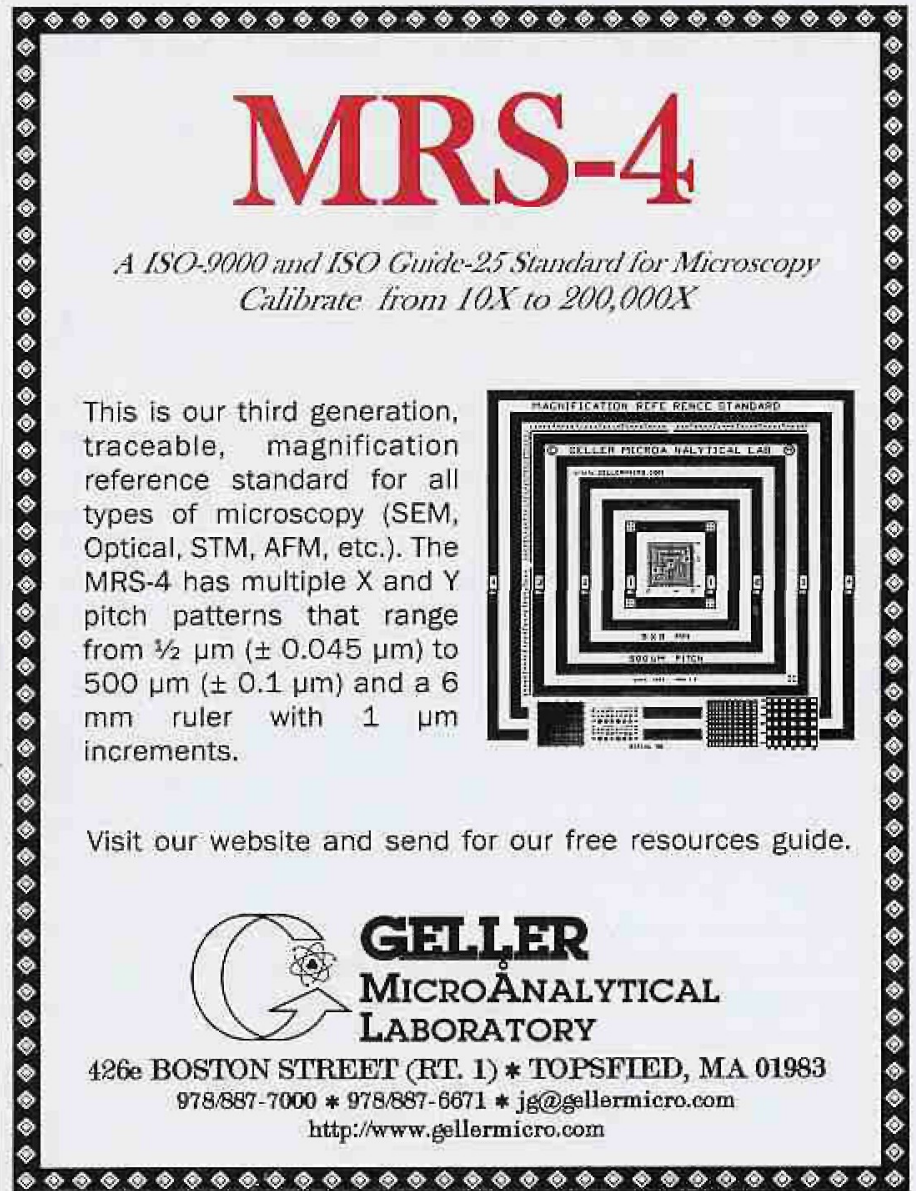

\section{Software to Make Your Job Easier, and a Free X-Checker Too!}

For a limited time, get a free $\mathrm{X}$-Checker calibration/performance standard (a $\$ 250$ value) when you purchase Electron Flight Simulator.

Electron Flight Simulator became the world's leading SEM/X-ray visualization software by showing you how your sample behaves under all conditions
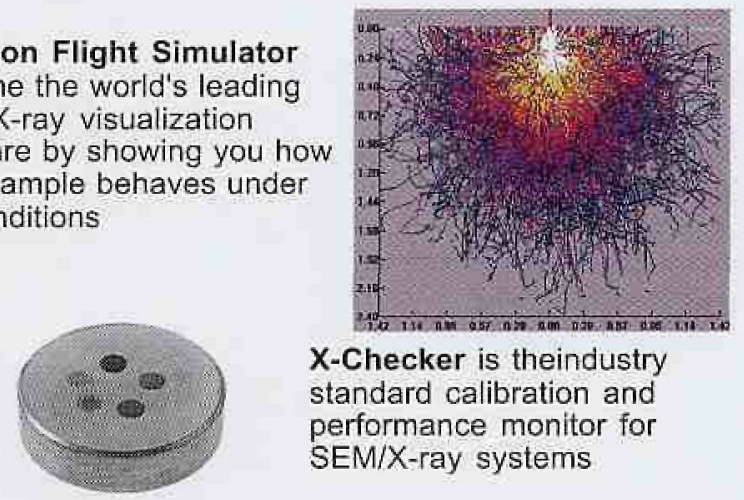

$\mathrm{X}$-Checker is theindustry standard calibration and performance monitor for SEM/X-ray systems

Put both to work in your lab and save $\$ 250$ ! www.small-world.net (703) 849-1492 


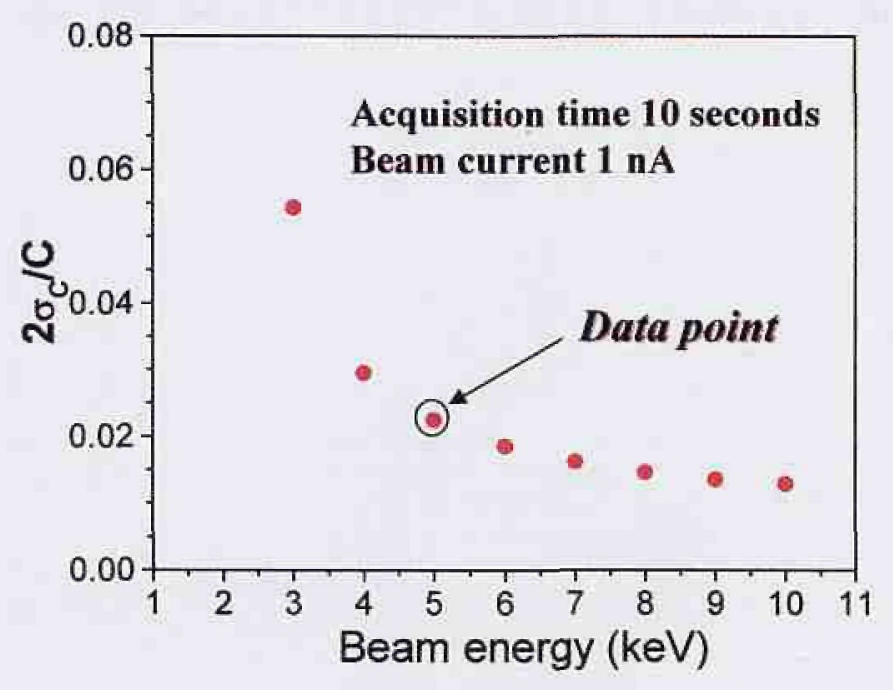

Figure $1 \mathrm{~b}$ The value of twice the relative standard deviation $2 \sigma / C$ vs. electron beam energy. The data point circled is the result obtain with the data shown in figure fa for ten repeat measurements on the sample and standard at a beam energy of $5 \mathrm{keV}$.

Furthermore, even if precision is not a factor, sources of systematic error such as beam drift and contamination could lead to errors far larger than those associated just with counting statistics. Based on the authors' experience, under good experimental measurement conditions, doubling the counting statistics based confidence interval might be a better estimate of overall variability, although this is admittedly somewhat arbitrary. Thus, the Al composition would be stated as $15.2 \pm 0.76$ weight percent for $95 \%$ confidence. Therefore, a reading of 17.0 weight percent from another point might be viewed as different, while one of 15.8 weight percent might be expected as a sample from the same distribution.

Figure $1 \mathrm{~b}$ serves to make several points. First, lowering the beam voltage and keeping other factors constant may be useful to improve spatial resolution by minimizing the $x$-ray excitation volume in a sample, but may cause a dramatic decrease in precision. Note at $2 \mathrm{keV} 2 \sigma / \mathrm{C}$ has increased to an estimated 0.08 . The second point is that the numbers presented here are for ten repeat measurement at 10 seconds each, which for the conditions used in this example was adequate time to obtain

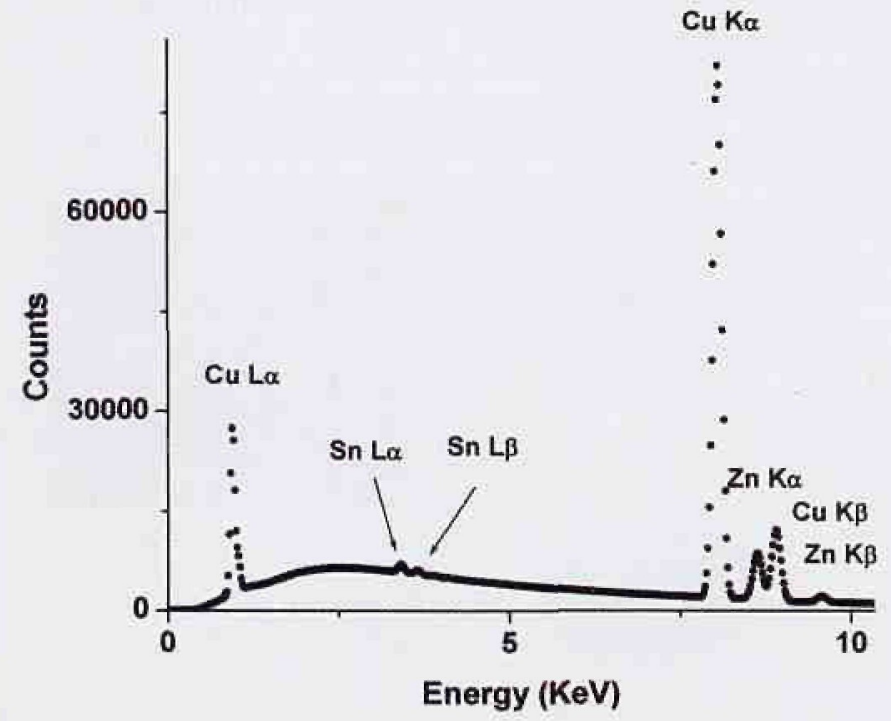

Figure 2a. Monte Carlo simulation of a Cu spectrum containing 9 weight percent $Z n$ and 1.0 weight percent $S n$. Conditions: 20 keV beam energy, $1 n A$ beam current, and a $40^{\circ}$ takeoff angle. The detector is a $6 \mathrm{~mm}$ Si(Li) placed $4 \mathrm{~mm}$ from the sample. Data collection time is 100 seconds.

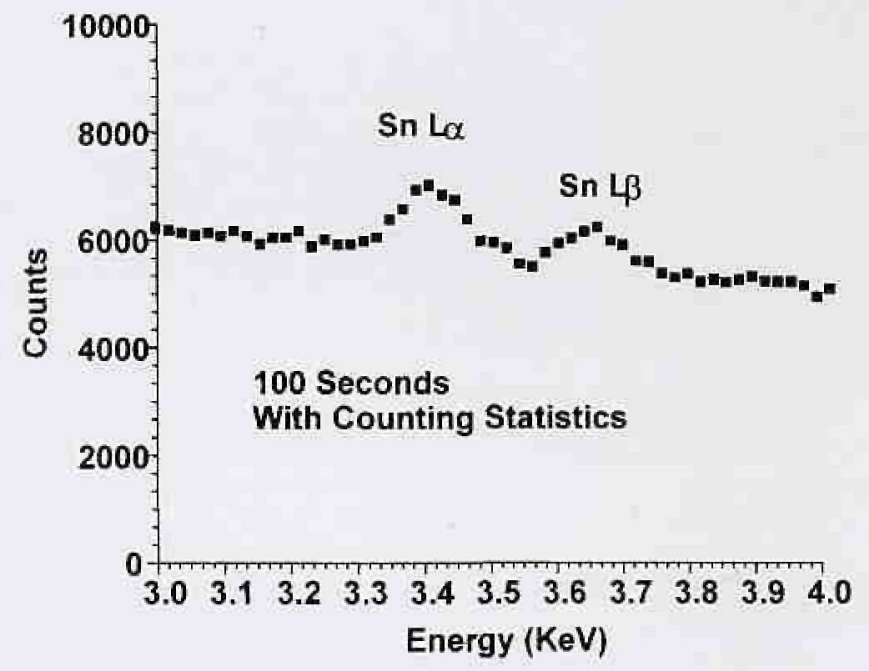

Figure $2 b$. Detail of Figure $2 b$ showing the $S n L$ peaks: a large number of counts on both the sample and standard. Since this may not always be the case, it is important to use equation (2) for each measurement made on your particular samples to determine if the value of $2 \sigma / C$ is good enough. $X$-ray microanalysis is capable of $2 \%$ or better accuracy, but if $2 \sigma_{c} / C$ is much larger than 0.02 , poor precision will mask good accuracy when two measurements are compared. Finally, it is also important to recognize that although the arsenic and aluminum peaks were close, there was not a serious overlap problem. Remember equation (2) requires background intensity data as well as peak intensity data. If the region of interest selected for a peak includes counts from an overlapping peak, then that should be included in the background counts in addition to any estimate of the background due to the $x$-ray continuum. If the peaks are very close; the precision may be unacceptable.

The determination of minimum detection limits was also discussed in detail in Zlebold's 1967 paper already cited (1). He concluded that the minimum detectability limit is given by:

$$
C_{D L} \geq 3.29 a /(n t P \cdot P / B)^{1 / 2}
$$

where " $a$ " is the parameter described in equation $(3), n$ is the number of repeat measurements, $t$ is the counting time, $P$ is the peak intensity on a pure elemental standard and $P / B$ is the peak to background ratio on that standard. This model uses a number of approximations that are necessary if the background on the actual sample is not known. However, a better estimate can be obtained if a standard with a detectable signal from the element of interest is available similar to the unknown, and also if the background on the unknown can be measured or approximated from the standard. In this case from reference (2):

$$
C_{D L} \geq \frac{3 \sqrt{\bar{N}(B)}}{\bar{N}_{S}-\bar{N}(B)} C_{S}
$$

where $\bar{N}(B)$ is the average value of the background on the unknown, $\bar{N}_{y}$ is the number of counts measured on the standard, and $\mathrm{C}_{s}$ is the concentration of the standard. Since a standard is not always available, Monte Carlo modeling of the spectrum of the standard can be of great help. Figure $2 a$ is a calculated spectrum for Cu containing 9 weight percent $Z n$ and 1.0 weight percent $S n$. Figure $2 b$ shows an expanded view of the region around the $S n L$ peaks. Data from this simulation entered into equation (5) gave a result of a detection limit for $S n$ of about 0.0522 weight percent for 100 seconds, $20 \mathrm{keV}$ beam energy, 1 nA beam current, $40^{\circ}$ takeoff angle, and a $6 \mathrm{~mm}$ Si $(\mathrm{Li})$ detector with $129 \mathrm{eV}$ located $4 \mathrm{~mm}$ from the sample. An advantage of the simulation approach is that it not only gives you a specific value for a detection limit, but it also allows for the adjustment of the model parameters. Thus different counting times, beam currents, beam voltages and other variables can be set to help you optimize the detection limit you need for a given problem.

In summary, it is suggested that concentration data be reported that 
shows not only the value measured, but also the confidence level associated with that value. While software packages sometimes include limits of error it is important to determine if they are based on equation (2). If not, all of the sources of variability based on $x$-ray counting statistics may not have been considered. Recognize that modeling techniques such as Monte Carlo calculation can be a very useful tool in estimating precision and detection limits even before a sample is ever placed in a microscope. Finally, also recognize that even so-called standardless analysis programs are based on some standard measurements and therefore, the variability of those standards should be taken into consideration in determining confidence limits. :

References

1. Ziebold, T. O. (1967), Anal. Chem. 39,848

2. Lifshin, E., Doganaksoy, N.,Sirois, J, and Gauvin, R. (1999), Microsc. Microanl, 4, 598-604

3. Goldstein, et. al. (2003), Scanning Electron Microscopy and X-ray Microanalysis, Kluwer Academic/Plenum Publishers

4. Ziebold, T. O. and R. E. Ogilvie (1964). Anal. Chem. 36, 322

5. Gauvin, R. and E.Lifshin, submitted to Microsc. Microanl.

\section{MICROSCOPIST BIOLOGY DEPARTMENT ST. LAWRENCE UNIVERSITY}

St. Lawrence University is seeking to fill a full-time, 12 month academic support staff position as a MICROSCOPY SPECIALIST. A master's degree or equivalent experience is required as is experience with confocal microscopy as well as electron microscopy (TEM and/or SEM). Mechanical and laboratory aptitude, computer experience, a desire to learn and teach new methodologies, and a positive work ethic is sought. The successful candidate will help oversee a developing interdisciplinary, multi-user microscopy/imagery center, will assist faculty and student researchers in advanced microscopy techniques, help teach microscopy methods and provide for the routine maintenance of the instrumentation infrastructure. The major instruments at the facility are on service contracts and the candidate will be expected to develop good working relationships with the professional service personnel. The facility is housed in the biology department and the successful candidate will also serve other science departments.

Applicants should send a letter of application, a current curriculum vitae (including references), a statement of any relevant research and teaching interests related to microscopy, and have three letters of recommendation forwarded to Dr. T. Budd, Biology Department, St. Lawrence University, Romoda Drive, Canton, NY 13617. The search committee will begin reviewing applications on July 21 and the search will remain open until filled.

We welcome applications from candidates who bring diverse cultural, ethnic and national perspectives to their creative work and teaching. St. Lawrence has a 10-year Master Plan that reflects support for excellence across the curriculum, a commitment to the quality and increased size of the faculty, a focus on well-balanced, learning-centered campus life and an historic investment in renovated and new facilities, all aimed to assure the University its place among the best institutions in the nation. For more information please visit SLU's homepage at http://www.stlawu.edu. St. Lawrence University is an Affirmative Action/Equal Employment Opportunity employer. Women, minorities, veterans, and persons with disabilities are encouraged to apply.

\section{Introducing the Max View Plus}

\section{Digital Camera Attachment System}

The MaxView Plus kit includes adapters to fit microscopes $\&$ optical devices with any of the following attachments; C-Mount, C/S-Mount, T-Mount, $23 \mathrm{~mm}$ Eyepiece Port, $30 \mathrm{~mm}$ Eyepiece Port, and $1.25^{\circ}$ Eyepiece Ports

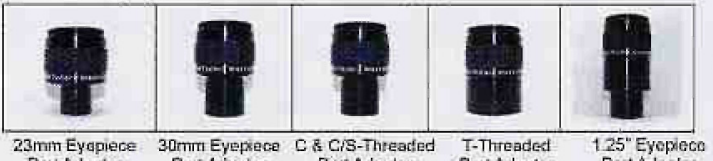

The MaxView Plus can also be used as a high quality wide ancla eyepiece With the included thread-an eyeguard. The MaxView Plus is " $T$ " threaded on top so you will need the appropriate Attactiment Kil to mate it to your particular digital camera. We carry dozens of attachment kits to fit most all populer digital cameras and we updete them regularly so your Maxview Plus will not become obsolete when it is time to upgracle to a new camera Plus will not become obsolete when it is time to upgrade to a new camera
if using it with a $35 \mathrm{~mm}$ SLR you will need the appropriate T-Ring lor your if using it with a $35 m$

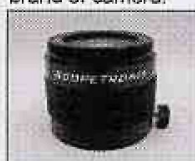

The heart of the MaxVlew system. A wide field $40 \mathrm{~mm}$ focal length four element symmetrical (Plossi) lens assembly specially mounted in a carefully designed custom cell. The lens is mounted only $1 \mathrm{~mm}$ from the top to mantain the close coupling required to reduce vipneting The cell features a unique sliding collar vigneltng. The cesl features a unue siling collar

it allows quick and easy removal of the cemera, it allows you to adjust eyeguard height for easy visual use, allows you to adjust magnification when used with a $35 \mathrm{~mm}$ camera, and allows you to adjust lens position to minimize vignetting with a digital camera.

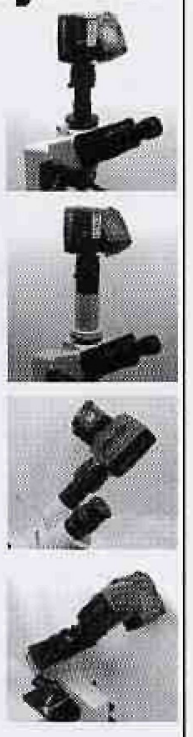

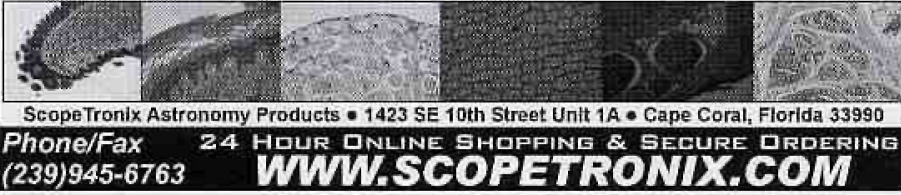

\section{Quantitative Image Analysis}

\section{with Dr. John C. Russ}

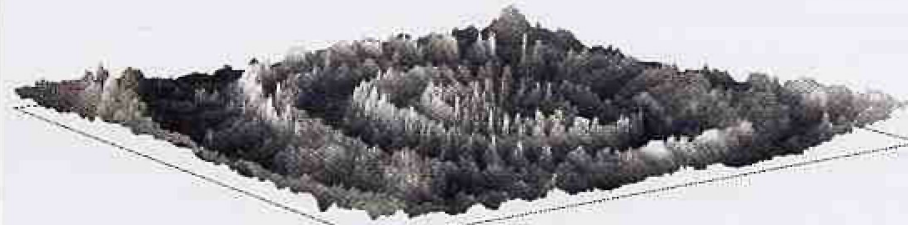

For more information including a detailed syllabus and course outline, see: http://www.reindeergraphics.com/courses

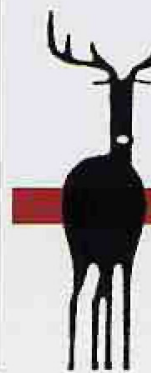

Currently scheduled courses: - San Francisco: Nov. 7-9, 2003

- Orlando: Mar. 11-12, 2004 